\title{
Estrutura fatorial do questionário de morbidade psiquiátrica de adultos aplicado em amostras populacionais de cidades brasileiras*
}

\author{
The factor structure of the adult psychiatry morbidity questionnaire (QMPA) in a \\ community sample of Brazilian cities
}

\author{
Sérgio Baxter Andreoli**, Jair de Jesus Mari**, Sérgio Luis Blay** Naomar de Almeida-Filho***, \\ Evandro Coutinho****, Josimar França*****, Jefferson Gomes Fernandes ${ }^{* * * * *}$, Ellis D'Arrigo

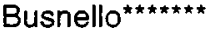

\begin{abstract}
ANDREOLI, S.B. et al. Estrutura fatorial do questionário de morbidade psiquiátrica de adultos aplicado em amostras populacionais de cidades brasileiras. Rev. Saúde Pública, 28: 249 - 60, 1994. A análise de componentes principais é uma técnica de estatística multivariada utilizada para examinar a interdependência entre variáveis. A sua principal característica é a capacidade de reduzir dados, e tem sido usada para o desenvolvimento de instrumentos de pesquisas psiquiátricas e na classificação dos transtornos psiquiátricos. Esta técnica foi utilizada para estudar a estrutura fatorial do Questionário de Morbidade Psiquiátrica do Adulto (QMPA). O questionário foi composto de 45 questões de resposta sim/não que identificam sintomas psiquiátricos, uso de serviço e de drogas psicotrópicas. O questionário foi aplicado em 6.470 indivíduos maiores de 15 anos, em amostras representativas da população de três cidades brasileiras (Brasília, São Paulo e Porto Alegre). O estudo teve como objetivo comparar a estrutura fatorial do questionário nas três regiões urbanas brasileiras. Sete fatores foram encontrados que explicam $42,7 \%$ da variância total da amostra. O fator 1, Ansiedade/Somatização ("eigenvalue" $(E V)=3.812$ e variância explicada $(\mathrm{VE})=10,9 \%) ; \mathrm{O}$ fator 2, Irritabilidade/Depressão $(\mathrm{EV}=2.412$ e $\mathrm{VE}=6,9 \%) ; \mathrm{O}$ fator 3, Deficiência Mental $(\mathrm{EV}=2.014 \mathrm{e} \mathrm{VE}=5,8 \%)$; $\mathrm{O}$ fator 4, Alcoolismo $(\mathrm{EV}=1.903 \mathrm{e} \mathrm{VE}=$ $5,4 \%) ; O$ fator 5, Exaltação do Humor $(E V=1.621$ e $V E=4,6 \%)$; $O$ fator 6 , Transtorno de Percep̧̧ão $(E V=1.599$ e VE $=4,6 \%)$ e o fator 7, Tratamento $(E V=1.592$ e VE $=4,5 \%) .0$ QMPA apresentou estruturas fatoriais semelhantes nas três cidades. Baseados nos achados, são feitas sugestões para que algumas questões sejam modificadas e para a exclusão de outras em uma futura versão do questionário.
\end{abstract}

Descritores: Psiquiatria, métodos. Escalas de graduação psiquiátrica. Análise fatorial.

\section{Introdução}

A natureza e a estrutura sintomatológica da morbidade psiquiátrica na comunidade têm sido crescentemente consideradas como importante tópico para pesquisas de cunho epidemiológico. Uma das estratégias dessa abordagem é aquela em que os dados são explorados em ordem para deter-

\footnotetext{
* Dissertação de Mestrado de Sérgio Baxter Andreoli apresentada à Escola Paulista de Medicina, 1993. Apresentada no 9 ith World Congress of Psychiatry na Paper Session: Psychiatric Epidemiology II, Rio de Janeiro, Brasil, 1993.

** Departamento de Psiquiatria e Psicologia Médica da Escola Paulista de Medicina, São Paulo, SP Brasil

*** Departamento de Medicina Preventiva da Universidade Federal de Bahia, Salvador, BA - Brasil

**** Departamento de Epidemiologia e Métodos Quantitativos em Saúde da Escola Nacional de Saúde Pública - Rio de Janeiro, RJ - Brasil
}

minar a sua estrutura, requerendo a aplicação de técnicas de análise multivariada (VázquezBarquero e col. ${ }^{22}, 1988$ ). O uso da análise fatorial e da análise de componentes principais para examinar dados coletados na comunidade através de escalas de rastreamento como o "General Health Questionnarie" (GHQ) (Goldberg9,10,11, 1972, 1978, 1990) e o "Psychiatric Epidemiology Re-

\footnotetext{
***** Departamento de Clínica Médica da Universidade de Brasília - Brasília, DF - Brasil

****** Departamento de Medicina Interna da Faculdade de Medicina da Pontifícia Universidade Católica do Rio Grande do Sul - Porto Alegre, RS - Brasil

******* Departamento de Psiquiatria e Medicina Legal da Faculdade de Medicina da Universidade Federal do Rio Grande do Sul - Porto Alegre, RS - Brasil
}

Separatas/Reprints: S.B. Andreoli - Rua Botucatu, $740-3^{\circ}$ andar 04023-900 - São Paulo, SP - Brasil

Edição subvencionada pela FAPESP. Processo 94/0500-0. 
search Interview" (PERI) (Dohrenwend e col.7, 1980) é um exemplo desta estratégia (Dohrenwend e col. ${ }^{8}, 1980$; D'Arcy ${ }^{6}, 1982$; Burvill e Knuiman ${ }^{4}$, 1983; Vázquez-Barquero e col. ${ }^{22}$, 1988). Esses estudos têm mostrado que existem transtornos psicopatológicos não específicos que podem ser identificados numa grande parcela da comunidade.

Diferente do GHQ e do PERI, que contêm questões inespecíficas para transtornos psiquiátricos, o Questionário de Morbidade Psiquiátrica do Adulto (QMPA) é questionário composto exclusivamente de questões que abrangem os mais freqüentes sinais e sintomas dos transtornos psiquiátricos, além de questões sobre tratamento psiquiátrico e uso de drogas psicofarmacológicas. O propósito do presente estudo é aplicar a técnica de análise fatorial para avaliar algumas propriedades psicométricas do QMPA, aplicado em amostras representativas da população de três cidades brasileiras (Brasilia, São Paulo e Porto Alegre), tendo como objetivo comparar as estruturas fatoriais entre as cidades.

\section{Metodologia}

A aplicação da análise de componentes principais foi feita com dados coletados em uma investigação populacional do tipo corte transversal $\mathrm{em}$ três áreas urbanas do Brasil. Foram utilizadas amostras representativas da população de adultos (idade acima de 15 anos), residentes nas cidades de Brasília, São Paulo e Porto Alegre. Os dados originaram-se das respostas obtidas com a aplicação de um questionário para levantamento de dados demográfico e um QMPA. Este último foi aplicado por entrevistador treinado para este fim, tendo como informante a dona da casa ou pessoa adulta adequada a prestar as informações (informante chave), respondendo também para todos os outros membros da família, maiores de 15 anos. Maiores detalhes da metodologia podem ser encontradas em Almeida-Filho e col. ${ }^{1}$ (1992).

\section{Plano de Amostragem}

Para o cálculo do tamanho das amostras, assumiu-se uma prevalência esperada de $10 \%$ de casos necessitados de cuidados psiquiátricos e um erro amostral de $2 \%$, com $95 \%$ de confiança. Este cálculo implicou o módulo de análise de 900 indivíduos que, mantendo a precisão das estimativas para até dois estratos, resultou em um tamanho amostral base de 1.800 sujeitos. Admitindo-se uma possibilidade de perdas e recusas de até $10 \%$ das amostras, estabeleceu-se em 2.000 o número de indivíduos que seriam submetidos à entrevista.
Amostragem do segmento Brasília: A amostragem realizada no Distrito Federal foi estratificada, considerando-se como estratos o plano piloto e as cidades satélites (Cruzeiro, Guará, Núcleo Bandeirante, Ceilândia, Brazilândia, Sobradinho, Planaltina, Gama, Tagualinga). De acordo com a distribuição demográfica, os estratos foram subdivididos em setores, segundo a situação socioeconômica da população. Dentro de cada setor, estabeleceu-se um número amostral de domicílios proporcional ao tamanho da sua população. Com base em um mapeamento atualizado, foi sorteado um domicílio como ponto de partida para cada entrevistador que prosseguia, incluindo na amostra a residência de número imediatamente superior, até esgotar um número de questionários previamente determinado pelos supervisores de campo.

Amostragem do segmento São Paulo: Foram selecionados três dos 48 subdistritos que compõem a área urbana de São Paulo (Brasilândia, Vila Guilherme e Aclimação), representando, respectivamente, os estratos socioeconômicos inferior, médio e superior, classificados de acordo com os critérios de Ramos e Goihman ${ }^{19}$ (1989). De modo a reproduzir a distribuição esperada dos estratos, selecionou-se aleatoriamente 17 setores censitários de Brasilândia, 8 de Vila Guilherme c 5 da Aclimação, que foram divididos em duas subáreas com vistas a um incremento na homogeneização. Em cada subárea, foratn sorteados 20 domicílios, localizados a partir do mapeamento atualizado pelo IBGE para o Censo-91 no caso de Brasilândia, e das listagens empregadas no Censo de 1980 , para os outros distritos.

Amostragem do segmento Porto Alegre: A regiâo metropolitana de Porto Alegre engloba 740 setores censitários, com uma média de 350 unidades habitacionais e 1.750 habitantes em cada setor. Está dividida em 96 zonas de tráfego que foram ordenadas segundo a renda média em 1986. Com essa informação, foram selecionados 10 estratos socioeconômicos, em cada um do estratos foram sorteados 3 domicílios que funcionaram como identificadores dos setores censitários incluídos na investigação. Dentro de cada setor censitário foram sorteados, então, 37 domicílios, sendo 30 para a amostra e 7 para eventuais reposições.

\section{Instrumento}

O QMPA foi construído baseado na experiência clínica de psiquiatras, no Cuestionario de Enfermedad Mental (Groot e Arevalo ${ }^{12}$ ) e no Questionário de Morbidade (Departamento de Medicina Preventiva da Universidade de São Pau$10^{21}, 1977$ ), sendo posteriormente desenvolvido e adaptado às especificidades regionais da lingua- 
gem por Santana ${ }^{20}$ (1982). O QMPA foi construído para ser um instrumento de rastreamento, sua validação, com dados populacionais, testado para o ponto de corte $6 / 7$, mostrou sensibilidade entre $75 \%$ e $93 \%$, especificidade entre $53 \%$ e $94 \%$ e Kappa ponderado de 0,88 .

A presente versão do QMPA contém 45 questões que abrangem os mais frequientes sinais e sintomas característicos de doenças mentais, além de questões sobre tratamento psiquiátrico, uso anterior $\mathrm{e}$ atual de drogas psicofarmacológicas. $\mathrm{O}$ questionário pode ser auto-aplicado ou aplicado por entrevistador e pode ser respondido por terceiros. As alternativas de respostas são $\operatorname{sim}$ ou $n \tilde{a} o$, às quais são atribuídos valores 1 ou 0 . As primeiras 43 quesiões devem ser respondidas referindo-se ao próprio indivíduo do qual se quer saber o estado de saúde mental. As duas últimas questões (questões 44 e 45 ) referem-se a sintomatologia apresentada por qualquer um dos membros da familia do indivíduo do qual se quer saber o estado de saúde mental.

O desempenho do QMPA na comunidade foi recentemente estudado por Almeida-Filho e col. ${ }^{1}$ (1992), através de sua validação. No presente estudo, o QMPA foi comparado com os resultados obtidos pela aplicação do Inventário de Sintoma do DSM-III (American Psychiatric Association ${ }^{2}$, 1980). Os indicadores de desempenho do QMPA mostraram-se muito abaixo dos obtidos em estudos anteriores, mostrando classificações incorretas acima de $33 \%$. A sensibilidade situou-se em torno de $50 \%$ e a especificidade variou de $70 \%$ a $80 \%$ para a identificação de qualquer nível de morbidade. $O$ QMPA apresentou valor preditivo positivo, aceitável em Brasília e Porto Alegre (em torno de $70 \%$ ) e fraco desempenho deste indicador em São Paulo (abaixo de $50 \%$ ). O valor preditivo negativo foi insatisfatório nas três cidades. Em síntese, o padrão de comportamento do QMPA foi o inverso do esperado para uma escala de rastreamento, ou seja, mostrou-se muito específico e pouco sensível.

\section{Análise}

Foram utilizadas para a análise as três matrizes de cada uma das cidades estudadas e a matriz contendo o somatório dos dados das três cidades (matriz total).

Foi calculado o quadrado da correlação múltipla (QCM) de cada questão com todas as outras para a identificação daquelas que apresentaram comportamento independente. Estas questões foram avaliadas separadamente. Foram construídas, também, matrizes reduzidas, nas quais foram retiradas as questões com QCM menor que 0,15.

A Análise de Componentes Principais (ACP) (Johnson e Wichern ${ }^{13}$, 1988; Laura ${ }^{18}, 1986$; Weiss ${ }^{23}, 1970$ ) foi aplicada nas matrizes de corre- lação produto-momento originadas das matrizes reduzidas e das matrizes não alteradas, nas três cidades e na amostra total. Para a determinação do número de fatores na $\mathrm{ACP}$, foi utilizado o critério de manter os fatores correspondentes aos "eigenvalues" da matriz maiores do que a unidade (Kai$\operatorname{ser}^{15}, 1960$ ). Para determinar a medida de confiabilidade dos fatores encontrados foi calculado o coeficiente Carmines' theta, que é um caso especial do coeficiente alpha de Cronbach's, derivado da análise de componentes principais (Carmines e Zeller $^{5}, 1979$ ). Depois de encontrada a matriz de cargas fatoriais, foi utjlizado o método de rotnção varimax (Kaiser ${ }^{14}$,1958). A matriz de cargas faioriais rodadas foi utilizada para a interpretação cos fatores. Os resultados foram comparados de modo descritivo entre as cidades e com os resultados ta análise da matriz total.

Para análise estatística foi utilizado o módulo $4 \mathrm{M}$ do pacote estatístico $\mathrm{BMDP}^{3}$ (1990).

\section{Consideraçōes sobre Análise de Componentes Principais}

A análise de componentes principais é uma técnica de análise multivariada que estuda a interdependência entre variáveis. Os objetivos mais importantes da análise de componentes principais são: gerar novas variáveis que possam expressar a informação contida em um conjunto original de dados; reduzir a dimensionalidade do problema que se está estudando, como passo para futuras análises; e eliminar, quando isso for possível, algumas variáveis originais se essas não trazem informação (Laura ${ }^{18}, 1986$ ). As novas variáveis geradas se denominam componentes principais, que são subconjuntos das variáveis originais altamente correlacionadas entre si, sendo que os subconjuntos possuem a propriedade estatística de independência.

Os parâmetros a serem obticios na análise são o Número de Fatores, Variância Explicada, Cargas Fatoriais. O Número de Fatores nos dá o número de dimensões (Fatores) formadas por um agrupamento de vetores no espaço vetorial (agrupamento de variáveis), que tem entre si uma relação de independência. Variância explicada, se cada Fator é uma composição de um subconjunto das variáveis originais e se este subconjunto pudesse ser usado como um substituto do conjunto original, qual seria sua capacidade de explicá-lo? A variância explicada é a medida da capacidade que um Fator tem de representar a variação total das variáveis originais e é dado como uma proporção. Cada variável tem com cada Fator uma correlação que mede a força com que esta variável está ligada a este Fator. A esta medida damos o nome de Carga Fatorial. Para que a variável seja considerada per- 
tencente a um determinado Fator e não a outro, o critério é arbitrário, entretanto, a maioria dos trabalhos utiliza como critério valores de cargas fatoriais acima de 0,40 ou 0,50 .

\section{Resultados}

No total foram aplicados 6.470 QMPAs nas três cidades pesquisadas. Em Brasília, 2.347; em São Paulo, 1.739; em Porto Alegre, 2.384. Não houve diferença entre os sexos nas três cidades.

A distribuição por idade nas três cidades (Tabela 1) mostrou que Braślia possui uma população mais jovem, com quase $60 \%$ abaixo de 35 anos. Por outro lado, Porto Alegre apresentou padrão oposto, com mais de $50 \%$ da população com idade acima de 35 anos. A distribuição de cada categoria de estado civil nas três cidades são semelhantes, apresentando em média $54,2 \%$ de solteiros, $35,5 \%$ de casados, $5,3 \%$ de viúvos e $6,4 \%$ de separados. A distribuição dessas categorias por sexo mostra mais mulheres entre os viúvos e separados.

As três cidades apresentaram padrões bastante diferentes nas proporções de naturalidade. Brasília apresentou no total $22 \%$ de pessoas naturais da cidade e $78 \%$ naturais de outros lugares. São Paulo mostra um equilíbrio, com $43,5 \%$ de pessoas naturais da cidade para $56,6 \%$ de pessoas de fora, das quais $15,9 \%$ são migrantes do próprio Estado. Porto Alegre apresentou padrão diferente das outras duas cidades com $38,6 \%$ das pessoas naturais da cidade, 53,3\% migrantes do Estado e apenas 8,5\% de pessoas de fora do Estado ou imigrantes.
A Tabela 2 mostra a distribuição quanto à forma de aplicação do QMPA. Chamou-se de informante ao próprio informante-chave, que respondeu o seu questionário e referido quando $o$ questionário foi respondido pelo informante-chave para um outro membro da família. Nas três cidades as mulheres responderam o QMPA como informantes e os homens tiveram seus QMPAs referidos. Tais diferenças foram estatisticamente significantes (Brasília X ${ }^{2}=397,9, \mathrm{df}=1$; São Paulo $X^{2}=149,5$, df $=1$; Porto Alegre $X^{2}=88,8$, $\mathrm{df}=1$; todos com $\mathrm{p}<0,001$ ).

\section{Questōes com Comportamento Independente}

Utilizando como critério o valor do QCM menor que 0,15 para considerar a questão como tendo um comportamento independente das outras questões do QMPA, as questões 12, 25, 26, 39 e 42 tiveram comportamento independente nas análises das amostras das três cidades. As questões 32, 44 e 45 somente em Brasília e a questão 17 somente em São Paulo e Porto Alegre. No modelo da amostra total tiveram este mesmo comportamento as questões $12,17,25,26,32,36,39,41,42$ e 45 .

\section{Fatores}

Foram encontrados 8 fatores em Brasília (Tabela 3), 11 em São Paulo (Tabela 4) e 9 em Porto Alegre (Tabela 5) que explicam, respectivamente, $45 \%, 52 \%$ e $47 \%$ da variância total da amostra de cada cidade. Na matriz total foram encontrados 7 fatores que explicam $42,7 \%$ da variância total da

Tabela 1 - Distribuição demográfica de sexo por idade em Brasília, São Paulo e Porto Alegre

\begin{tabular}{|c|c|c|c|c|c|c|}
\hline \multirow{2}{*}{$\frac{\text { Cidade }}{\text { Idade/sexo }}$} & \multicolumn{2}{|c|}{ Brasília } & \multicolumn{2}{|c|}{ São Paulo } & \multicolumn{2}{|c|}{ Porto Alegre } \\
\hline & Mas $\%$ & Fem \% & Mas \% & Fem \% & Mas $\%$ & Fem \% \\
\hline $\begin{array}{l}15-24 \\
25-34 \\
35-44 \\
45-44 \\
55-\end{array}$ & $\begin{array}{l}375(34,5) \\
255(23,5) \\
194(17,9) \\
149(13,7) \\
113(10,4)\end{array}$ & $\begin{array}{l}427(33,9) \\
329(26,1) \\
218(17,3) \\
152(12,1) \\
134(10,6)\end{array}$ & $\begin{array}{l}242(28,6) \\
227(26,9) \\
136(16,1) \\
112(13,3) \\
128(15,1)\end{array}$ & $\begin{array}{l}217(24,3) \\
226(25,2) \\
150(16,8) \\
134(15,0) \\
167(18,7)\end{array}$ & $\begin{array}{l}275(25,5) \\
235(21,8) \\
215(20,0) \\
140(13,0) \\
213(19,8)\end{array}$ & $\begin{array}{l}276(21,1) \\
277(21,2) \\
254(19,4) \\
202(15,4) \\
300(22,9)\end{array}$ \\
\hline Total & $1.086(100)$ & $1.260(100)$ & $845(100)$ & $894(100)$ & $1.075(100)$ & $1.309(100)$ \\
\hline
\end{tabular}

Tabela 2 - Distribuição da forma de aplicação do QMPA na três cidades por sexo

\begin{tabular}{|c|c|c|c|c|c|c|}
\hline \multirow{2}{*}{$\frac{\text { Cidade }}{\text { Forma/sexo }}$} & \multicolumn{2}{|c|}{ Brasilia } & \multicolumn{2}{|c|}{ São Paulo } & \multicolumn{2}{|c|}{ Porto Alegre } \\
\hline & Mas \% & Fem \% & Mas \% & Fem \% & Mas \% & Fem \% \\
\hline $\begin{array}{l}\text { Informante } \\
\text { Referido }\end{array}$ & $\begin{array}{l}178(20,0) \\
909(62,3)\end{array}$ & $\begin{array}{l}711(80,0) \\
549(37,7)\end{array}$ & $\begin{array}{l}163(28,0) \\
682(59,0)\end{array}$ & $\begin{array}{l}420(72,0) \\
474(41,0)\end{array}$ & $\begin{array}{l}294(32,7) \\
781(52,6)\end{array}$ & $\begin{array}{l}604(67,3) \\
705(47,4)\end{array}$ \\
\hline Total & $1.087(46,3)$ & $1.260(53,7)$ & $845(48,6)$ & $894(51,4)$ & $1.075(45,1)$ & $1.309(54,9)$ \\
\hline
\end{tabular}


Tabela 3 - Fatores resultantes da análise do componente principal da matriz reduzida, Brasilia

\begin{tabular}{|c|c|}
\hline Fator $1-E V=3.023-V E=8,3 \%$ & Cargas \\
\hline $\begin{array}{l}\text { 05. Sente fraqueza nas pernas, dores nos nervos? } \\
\text { 04. Sente dores ou pontadas freqüentes na cabeça? } \\
\text { 03. Se queixa de zumbidos nos ouvidos, agonia na cabeça ? } \\
\text { 11. Tem dificuldade de aprender, lembrar ou entender coisas ? } \\
\text { 08. Sente bolo na garganta, queimação ou empachamento no estómago ? } \\
\text { 09. Sente tremores ou frieza nas mãos? } \\
\text { 21. Se queixa de palpitação ou aperto no coração? } \\
\text { 07. Fica periodos triste, com desânimo? }\end{array}$ & $\begin{array}{l}0.659 \\
0.573 \\
0.554 \\
0.525 \\
0.516 \\
0.483 \\
0.435 \\
0.409\end{array}$ \\
\hline
\end{tabular}

Fator $2 \cdot E V=2.57-V E=6,9 \%$

Irritabilidade/Depressão

10. Tem com freqüência crises de irritação?

06. Fica agressivo, explode com facilidade?

22. Sofre de nervosismo ou está sempre intranqüilo?

18. Fica fechado no quarto sem querer ver ninguém ?

0.604

14. Já pensou em dar fim na vida ?

15. Já esteve descontrolado, fora de si, como se fosse doente da cabeça?

Fator $3-E V=2.003-V E=5,5 \%$

Deficiência Mental

38. Sofre de retardamento mental ?

37. Sofre de acesso de loucura?
36. Não consegue freqüentar a escola ?

16. Não consegue trabalhar por nervosismo ou doença mental ?

Fator $4 \cdot E V=1.977 \cdot V E=5,4 \% \quad$ Alcoolismo

19. Se embriaga pelo menos uma vez por semana? $\quad 0.806$

43. Bebe exageradamente?

0.805

20. Bebe diariamente?

0.768

Fator $5-E V=1.893-V E=5,2 \%$

Tratamento

35. Já utilizou ou usa atualmente remédio para dormir ou acalmar os nervos ?

40. Recebe tratamento para nervosismo ou doença mental ?

02. Tem dificuldade para dormir?

Fator $6-E V=1.811-V E=4,9 \% \quad$ Exaltação do Humor

33. Fica períodos exageradamente alegre sem saber por quê ?

29. Fala ou ri sozinho?

34. Fica andando muito, cantando ou falando sem parar ?

28. Fala coisas sem sentido, bobagens?

0.565

Fator $7-E V=1.664-V E=4,5 \%$

Conversão/Convulsão

24. Já sofreu um ataque depois de um susto ou contrariedade?

17. Já ficou sem falar ou enxergar ?

41. Sofre de ataques, caindo no chão se batendo ?

Fator $8-E V=1.543-V E=4,3 \%$

Transtorno de Percepção

31. Sente que está sendo controlado por telepatia, por rádio ou espírito ?

30. Se acha perseguido, que estão querendo the fazer mal ?

27. Se queixa de ouvir vozes ou vê coisas que os outros não vêem ?

$E V=$ "eigenvalue"

VE = variância explicada

amostra (Tabela 6). Os coeficientes theta foram, respectivamente, $0,86,0,85,0,87$ e 0,86 .

Os fatores encontrados em Brasília foram: Ansiedade/Somatização ("eigenvalues" $(E V)=3.023$ e Variância Explicada (VE) $=8,3 \%$ ); Irritabilidade/Depressão $(E V=2.57$ e VE $=6,9 \%)$; Defi- ciência Mental $(\mathrm{EV}=2.003$ e VE $=5.5 \%)$; Alcoolismo $(E V=1.977$ e VE $=5,4 \%)$; Tratamento (EV $=1.893$ e VE $=5,2 \%)$; Exaltação do Humor $(\mathrm{EV}=$ 1.811 e VE $=4,9 \%$ ); e Conversão/Convulsão (EV $=1.664$ e VE $=4,5 \%$ ) e o Transtorno de Percepção $(\mathrm{EV}=1.543 \mathrm{e} \mathrm{VE}=4,3 \%)$. 
Tabela 4 - Fatores resultantes da análise do componente principal da matriz reduzida, São Paulo

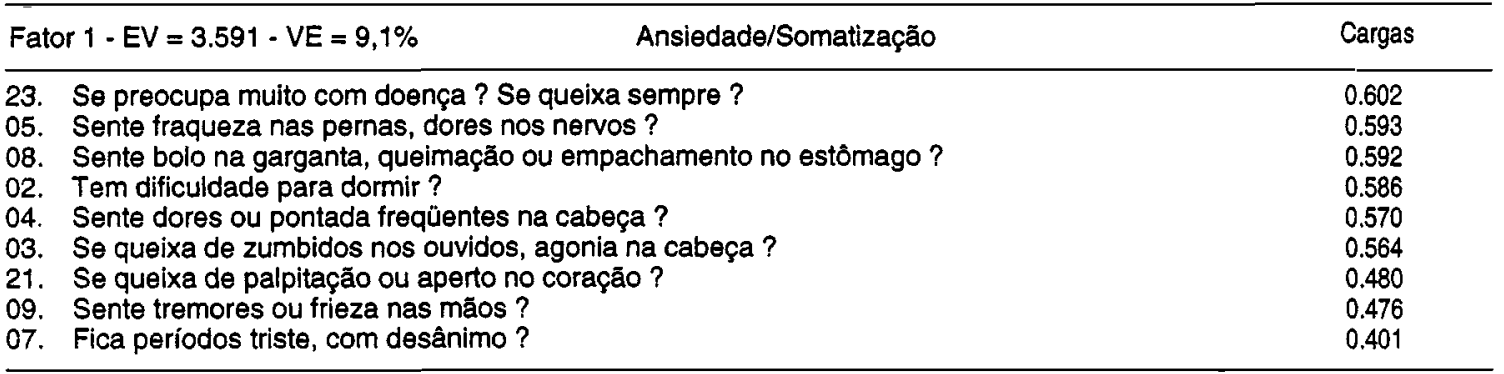

Fator $2 \cdot E V=2.147-V E=5,4 \%$

Irritabilidade

10. Tem com freqüência crises de irritação ?

06. Fica agressivo, explode com facilidade?

22. Sofre de nervosismo ou está sempre intranqüilo ?

07. Fica períodos triste, com desânimo?

Fator $3-E V=2.031-V E=5,2 \% \quad$ Alcoolismo

43. Bebe exageradamente ?

19. Se embriaga pelo menos uma vez por semana?

20. Bebe diariamente?

Fator $4 \cdot E V=1.801-V E=4,6 \%$

Descontrole Emocional

37. Sofre de acesso de loucura?

16. Não consegue trabalhar por nervosismo ou doença mental ?

15. Já esteve descontrolado, fora de si, como se fosse doente da cabeça ?

Fator $5-E V=1.737-V E=4,4 \%$

Antecedentes Familiares

44. Não sabe se vestír, urina ou defeca nas roupas?

45. Não fala, não caminha, não reconhece as pessoas ?

Fator $6-E V=1.635 \cdot V E=4,1 \%$

Transtornos de Percepção

31. Sente que está sendo controlado por telepatia, por rádio ou espirito ?

27. Se queixa de ouvir vozes ou vê coisas que os outros não vêem?

30. Se acha perseguido, que estão querendo the fazer mal ?

Fator $7 \cdot E V=1.630 \cdot V E=4,1 \% \quad$ Depressão

18. Fica fechado no quarto sem querer ver ninguém ?

13. As vezes fica parado, chorando muito?

Fator $8-E V=1.619-V E=4.1 \%$

Exaltação do Humor

34. Fica andando muito, cantando ou falando sem parar ?

33. Fica períodos exageradamente alegre sem saber por quê ?

32. Às vezes fica muito tempo numa posição estranha?

Fator $9-E V=1.490 \cdot$ VE $=3,8 \%$

Tratamento

40. Recebe tratamento para nervosismo ou doença mental ?

35. Já utilizou ou usa atualmente remédio para dormir ou acalmar os nervos ?

32. Ás vezes fica muito tempo numa posição estranha?

Fator 10 - EV $=1.463-$ VE $=3,7 \%$

Transtorno de Aprendizagem

36. Não consegue freqüentar a escola ?

11. Tem dificuldades de aprender, lembrar ou entender coisas?

0.409

38. Sofre de retardamento mental ?

Fator $11-E V=1.369-$ VE $=3,5 \% \quad$ Conversão/Convulsão

41. Sofre de ataques, caindo no chão se batendo ?

24. Já sofreu um ataque depois de um susto ou contrariedade? 
Tabela 5 - Fatores resultantes da análise do componente principal da matriz reduzida, Porto Alegre

\begin{tabular}{|c|c|c|}
\hline Fator & $1-E V=4.044-V E=10,4 \%$ & Cargas \\
\hline $\begin{array}{l}03 . \\
02 . \\
\text { 21. } \\
05 . \\
04 . \\
\text { 35. } \\
\text { 22. } \\
08 . \\
09 \\
07 . \\
\text { 23. } \\
11 .\end{array}$ & $\begin{array}{l}\text { Se queixa de zumbidos nos ouvidos, agonia na cabeça? } \\
\text { Tem diiculdade para dormir ? } \\
\text { Se queixa de palpitação ou aperto no coração? } \\
\text { Sente fraqueza nas pernas, dores nos nervos ? } \\
\text { Sente dores ou pontadas freqũentes na cabeça ? } \\
\text { Já utilizou ou usa atualmente remédio para dormir ou acalmar os nervos ? } \\
\text { Sofre de nervosismo ou está sempre intranqüilo? } \\
\text { Sente bolo na garganta, queimação ou empachamento no estômago? } \\
\text { Sente tremores ou frieza nas mãos? } \\
\text { Fica periodos triste, com desânimo? } \\
\text { Se preocupa muito com doença ? Se queixa sempre? } \\
\text { Tem dificuldade de aprender, lembrar ou entender coisas? }\end{array}$ & $\begin{array}{l}0.619 \\
0.617 \\
0.601 \\
0.599 \\
0.554 \\
0.534 \\
0.514 \\
0.496 \\
0.491 \\
0.485 \\
0.471 \\
0.406\end{array}$ \\
\hline
\end{tabular}

Fator $2 \cdot E V=2.412-V E=6,2 \%$

Irritabilidade/Depressão

10. Tem com freqũência crises de irritação?

06. Fica agressivo, explode com facilidade ?

18. Fica fechado no quarto sem querer ver ninguém ?

13. Às vezes fica parado, chorando muito ?

14. Já pensou em dar fim na vida?

07. Fica períodos triste, com desânimo?

Fator $3-E V=2.081-V E=5,4 \%$

Deficiência Mental

38. Sofre de retardamento mental ?

36. Não consegue freqüentar a escola ?

44. Não sabe se vestir, urina ou defeca nas roupas?

37. Sofre de acesso de loucura ?

16. Não consegue trabalhar por nervosismo ou doença mental ?

Fator $4-E V=1.834-V E=4,7 \%$

Tratamento

40. Recebe tratamento para nervosismo ou doença mental ?

35. Já utilizou ou usa atualmente remédio para dormir ou acalmar os nervos ?

16. Não consegue trabalhar por nervosismo ou doença mental ?

15. Já esteve descontrolado, fora de si, como se fosse doente da cabeça ?

Fator $5-E V=1.806-V E=4,6 \%$

Alcoolismo

43. Bebe exageradamente?

19. Se embriaga pelo menos uma vez por semana?

20. Bebe diariamente?

Fator $6 \cdot E V=1.680 \cdot V E=4,3 \%$

Transtorno Psicomotor

41. Sofre de ataques, caindo no chão se batendo ?

32. Às vezes fica muito tempo numa posição estranha?

34. Fica andando muito, cantando ou falando sem parar ?

Fator $7-E V=1.64 \uparrow-V E=4,2 \%$

Transtorno de Percepção

27. Se queixa de ouvir vozes ou vê coisas que os outros não vêem ?

31. Sente que está sendo controlado por telepatia, por rádio ou espírito ?

Fator $8-E V=1.492-V E=3,8 \%$

Comportamento Bizarro

29. Fala ou ri sozinho ?

01. Sofre de falta de apetite ?

28. Fala coisas sem sentido, bobagens?

Fator $9-E V=1.314-V E=3,4 \%$

Antecedentes Familiares

45. Não fala, não caminha, não reconhece as pessoas ?

44. Não sabe se vestir, urina ou defeca nas roupas?

24. Já sofreu um ataque depois de um susto ou contrariedade ?

$\mathrm{EV}=$ "eigenvalue"

$\mathrm{VE}=$ variância expliçada 
Tabela 6 - Fatores resultantes da análise do componente principal da matriz reduzida, Amostra Total

\begin{tabular}{|c|c|c|}
\hline Fator & $1-E V=3.812-V E=10,9 \%$ & Cargas \\
\hline 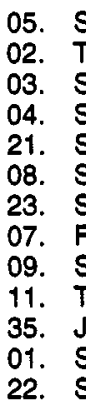 & $\begin{array}{l}\text { Sente fraqueza nas pernas, dores nos nervos ? } \\
\text { Tem dificuldade para dormir? } \\
\text { Se queixa de zumbidos nos ouvidos, agonia na cabeça? } \\
\text { Sente dores ou pontadas freqũentes na cabeça? } \\
\text { Se queixa de palpitação ou aperto no coração? } \\
\text { Sente bolo na garganta, queimação ou empachamento no estômago? } \\
\text { Se preocupa muito com doença? Se queixa sempre? } \\
\text { Fica períodos triste, com desânimo? } \\
\text { Sente tremores ou frieza nas mãos? } \\
\text { Tem dificuldade de aprender, lembrar ou entender coisas? } \\
\text { Já utilizou ou usa atualmente remédio para dormir ou acalmar os nervos? } \\
\text { Sofre de falta de apetite? } \\
\text { Sofre de nervosismo ou está sempre intranqüilo? }\end{array}$ & $\begin{array}{l}0.618 \\
0.604 \\
0.592 \\
0.577 \\
0.529 \\
0.519 \\
0.499 \\
0.472 \\
0.463 \\
0.431 \\
0.430 \\
0.425 \\
0.410\end{array}$ \\
\hline
\end{tabular}

Fator $2 \cdot E V=2.412-V E=6,9 \%$

Irritabilidade/Depressão

10. Tem com freqüência crises de irritação ?

06. Fica agressivo, explode com facilidade?

18. Fica fechado no quarto sem querer ver ninguém ?

22. Sofre de nervosismo ou está sempre intranqüilo ?

14. Já pensou em dar fim na vida?

13. As vezes fica parado, chorando muito?

15. Já esteve descontrolado, fora de si, como se fosse doente da cabeça?

07. Fica períodos triste, com desânimo?

Fator $3-E V=2.014-V E=5,8 \%$

Deficiência Mental

38. Sofre de retardamento mental ?

44. Não sabe se vestir, urina ou defeca nas roupas ?

37. Sofre de acesso de loucura?

0.576

16. Não consegue trabalhar por nervosismo ou doença mental ?

0.567

Fator $4-E V=1.903-V E=5,4 \% \quad$ Alcoolismo

43. Bebe exageradamente?

19. Se embriaga pelo menos uma vez por semana?

20. Bebe diariamente?

Fator $5-E V=1.621-V E=4,6 \%$

Exaltação do Humor

33. Fica períodos exageradamente alegre sem saber por quê ?

34. Fica andando muito, cantando ou falando sem parar ?

29. Fala ou ri sozinho?

28. Fala coisas sem sentido, bobagens?

Fator $6 \cdot E V=1.599-V E=4,6 \%$

Transtorno de Percepção

31. Sente que está sendo controlado por telepatia, por rádio ou espirito ?

27. Se queixa de ouvir vozes ou vé coisas que os outros não vêem ?

30. Se acha perseguido, que estão querendo the fazer mal ?
Fator $7-E V=1.592-V E=4,5 \%$
Tratamento

40. Recebe tratamento para nervosismo ou doença mental ?

35. Já utilizou ou usa atualmente remédio para dormir ou acalmar os nervos ?

24. Já sofreu um ataque depois de um susto ou contrariedade?

Os fatores encontrados em São Paulo foram: Ansiedade/Somatização ("eigenvalues" $=3.591 \mathrm{e}$ Variância Explicada $=9,1 \%)$; Irritabilidade (EV = 2.147 e VE $=5,4 \%$ ); Alcoolismo (EV $=2.031$ e VE $=5,2 \%) ;$ Descontrole Emocional $(E V=1.801$ e VE $=4,6 \%)$; Antecedentes Familiares $(E V=1.737 \mathrm{e}$ VE
$=4,4 \%)$; Transtorno de Percepção (EV $=1.635$ e VE $=4,1 \%)$; Depressão $(\mathrm{EV}=1.630$ e VE $=4,1 \%)$; Exaltação do Humor $(E V=1.619$ e VE $=4,1 \%)$; Tratamento $(E V=1.490$ e VE $=3,8 \%)$; Transtorno de Aprendizagem (EV $=1.463$ e VE $=3,7 \%)$ e o Conversão/Convulsão $(\mathrm{EV}=1.369 \mathrm{e} \mathrm{VE}=3,5 \%)$. 
Os fatores encontrados em Porto Alegre foram: Ansiedade/Somatização ("eigenvalues" $=4.044 \mathrm{e}$ Variância Explicada $=10,4 \%$ ); Irritabilidade/ Depressão $(E V=2.412$ e VE $=6,2 \%$ ); Deficiência Mental $(E V=2.081$ e VE $=5,4 \%)$; Tratamento $(\mathrm{EV}=1.834$ e $\mathrm{VE}=4,7 \%)$; Alcoolismo $(\mathrm{EV}=$ 1.806 e VE $=4,6 \%$ ); Transtorno Psicomotor (EV $=1.680$ e VE $=4,3 \%$ ); Transtorno de Percepção $(E V=1.641$ e VE $=4,2 \%)$; Comportamento Bizarro ( $E V=1.492$ e VE $=3,8 \%)$ e o Antecedentes Familiares ( $\mathrm{EV}=1.314 \mathrm{e} \mathrm{VE}=3,4 \%$ ).

Os fatores encontrados na amostra total foram: Ansiedade/Somatização ("eigenvalues" $=3.812 \mathrm{e}$ Variância Explicada = 10,9\%); Irritabilidade/ Depressão (EV $=2.412$ e VE $=6,9 \%$ ); Deficiência Mental $(E V=2.014$ e $V E=5,8 \%)$; Alcoolismo $(E V=1.903$ e VE $=5,4 \%)$; Exaltação do Humor $(E V=1.621$ e VE $=4,6 \%)$; Transtorno de Percepção $(E V=1.599$ e VE $=4,6 \%)$ e o Tratamento $(\mathrm{EV}=1.592$ e $\mathrm{VE}=4,5 \%)$.

\section{Discussão}

Algumas questões do QMPA tiveram comportamento independente, revelado pelos baixos valores do quadrado da correlação múltipla. Observando o comportamento dessas questões nas matrizes não alteradas, analisadas pela ACP, algumas mostram parte da sua variabilidade associada a quadros psicopatológicos pertinentes aos seus conteúdos.

Entretanto, o comportamento independente mostra uma outra parte desta variabilidade não necessariamente patologica. Tal foi o caso das questões $12,17,26,32$ e 39 . Outras questões, também com comportamento independente, não são ambíguas e mostram relação direta com o quadro psicopatológico. Tal foi o caso das questões 25,41 e 42. As questões 44 e 45 também mostraram relação direta com o quadro psicopatológico, mas apresentaram problemas ao serem respondidas, por serem referidas a um membro da família.

Os fatores resultantes das análises das matrizes reduzidas das três cidades apresentaram muitas semelhanças, o que nos permitiu considerar o resultado da análise da matriz total como representante dos fatores encontrados em cada uma das cidades em separado, são eles:

Ansiedade/Somatização $(\mathrm{EV}=3.812, \mathrm{VE}=$ $10,9 \%$ ). Este fator representa a dimensão psicopatológica mais estável, em todas as cidades. Em todos os métodos utilizados, sempre aparece como primeiro fator, explicando uma parte importante da variância total das amostras. O fator foi assim chamado porque sempre agrupa os sintomas 3 ("Se queixa de zumbidos nos ouvidos, agonia na cabeça?"), 4 ("Sente dores ou pontadas freqüentes na cabeça?"), 5 ("Sente fraqueza nas pernas, dores nos nervos?") e 8 ("Sente bolo na garganta, queimação ou empachamento no estômago?") relacionados com somatização, e os dois sinais de disfunção somática, 9 ("Sente tremores ou frieza nas mãos?"), e 21 ("Se queixa de palpitação ou aperto no coração?"), relacionados a perturbações fisiológicas associadas à ansiedade. Estão também sempre associados à questão 7 ("Fica períodos triste, com desânimo?") relacionada a um estado de humor depressivo.

Conforme vai aumentando a idade da população, de Brasília para Porto Alegre, o sintoma de insônia 2 ("Tem dificuldade para dormir?"), talvez mais apropriadamente chamado de transtorno do sono no caso do idoso (Kaplan e Sadock $^{16}, 1990$ ), vai ganhando importância. Não aparece em Brasília e aparece em São Paulo e Porto Alegre. Nesta última, aparece com a maior carga fatorial e associado à questão 35 ("Já utilizou ou usa atualmente remédio para dormir ou acalmar os nervos?") relacionada a tratamento de transtorno do sono.

Irritabilidade/Depressão $(\mathrm{EV}=2.412, \mathrm{VE}=$ $6,9 \%$ ). Este fator já não apresenta a mesma estabilidade encontrada no fator anterior, exceto pelas questões 10 ("Tem com freqüência crises de irritação?") e 6 ("Fica agressivo, explode com facilidade?"), que refletem estado de humor irritável.

Associadas a este fator encontramos, as questões 18 e 14 em Brasília e as questões 7, 13, $14 \mathrm{e} 18 \mathrm{em}$ Porto Alegre, questões que refletem estado de humor depressivo. Em São Paulo, somente a questão 7 ("Fica períodos triste, com desânimo?") está associada a esta dimensão; as outras questões, 18 ("Fica fechado no quarto sem querer ver ninguém?") e 13 ("Às vezes fica parado, chorando muito?"), relacionadas a humor depressivo, vão formar um fator específico (Fator $7 /$ Depressão). Se pré-estabelecemos um número de fatores igual a 9, na ACP de São Paulo, encontramos um fator Irritabilidade/Depressão semelhante àqueles encontrados nas outras cidades e o fator Depressão desaparece.

Estes achados mostram que esta dimensão psicopatológica é consistente na comunidade, mostrando uma estreita correlação entre a irritabilidade e a depressão, e não uma correlação entre irritabilidade e os estados de exaltação do humor. Aliás, como será visto mais adiante, os sinais que refletem estado de exaltação do humor formam um fator próprio, evidenciando outras associações.

Deficiência Mental $(\mathrm{EV}=2.014, \mathrm{VE}=5,8 \%)$. Este fator é muito bem definido em Brasília e Porto Alegre, apresentando a questão 38 ("Sofre de retardamento mental?") com a maior carga fatorial. 
Esta questão sempre aparece associada às questões 16 ("Não consegue trabalhar por nervosismo ou doença mental?"), 36 ("Não consegue freqüentar a escola?") e 37 ("Sofre de acesso de loucura?"), que refletem uma vulnerabilidade a transtornos mentais dos pacientes com deficiência mental leve ou moderada (Kaplan e Sadock $\left.{ }^{16}, 1990\right)$. Essas questões estariam refletindo a manifestação de defesas do paciente deficiente contra um intolerável senso de inadequação, baixa auto-estima e ansiedade. Estas defesas podem ser decorrentes de situações patológicas ou de má adaptação, levando a um comportamento inadequado, sentimentos de inadequação, frustração e reações agressivas.

Alcoolismo $(\mathrm{EV}=1.903, \mathrm{VE}=5,4 \%)$. É representado pelas questões 19 ("Se embriaga pelo menos uma vez por semana?"), 20 ("Bebe diariamente?") e 43 ("Bebe exageradamente?"); é um fator onde as cargas possuem os maiores valores, mostrando a alta correlação destas questões com esta dimensão psicopatológica. É também a dimensão que menos deixa dúvida quanto a seu caráter patológico.

Exaltação do Humor (EV = 1.621, $\mathrm{VE}=4,6 \%$ ). As questões 33 ("Fica períodos exageradamente alegre sem saber por quê?") e 34 ("Fica andando muito, cantando ou falando sem parar?") são as principais questões deste fator. Aparecem sempre juntas, exceto na ACP da matriz reduzida de Porto Alegre, onde a questão 33 não obteve carga fatorial $(0,356)$ suficiente para integrar o fator. No restante das análises formam juntas a dimensão Exaltação do Humor, associadas às questões 28 ("Fala coisas sem sentido, bobagens?") e 29 ("Fala ou ri sozinho?").

No comportamento das questões 28 e 29 vamos observar que: em Brasília, toda a carga fatorial está concentrada no fator Exaltação de Humor; em São Paulo, aparecem marginalmente associadas ao fator Exaltação do Humor, com cargas fatoriais de 0,340 e 0,311 respectivamente aparecendo também associadas marginalmente com o fator Descontrole Emocional, com cargas de 0,368 e 0,316; em Porto Alegre, juntas, formam um fator que denominamos Comportamento Bizarro e associa as questões $33 \mathrm{e}$ 34 marginalmente com cargas fatoriais de 0,333 e 0,327 . Na amostra total, questão 28 aparece associada ao fator Exaltação do Humor e, marginalmente, ao fator Deficiência Mental, com carga fatorial de 0,339, ao passo que a questão 29 só aparece associada ao fator Exaltação do Humor.

Estas observações mostram que as questões 33 e 34 definem esta dimensão e a maior parte da variabilidade das questões 28 e 29 está associada também a esta dimensão psicopatológica. Provavelmente estas alterações de comportamento estão representando um diagnóstico de mania.
Transtornos da Percepção $(\mathrm{EV}=1.599, \mathrm{VE}=$ $4,6 \%$ ). É representado pelas questões 27 ("Se queixa de ouvir vozes ou vê coisas que os outros não vêem?"), 30 ("Se acha perseguido, que estão querendo the fazer mal?") e 31 ("Sente que está sendo controlado por telepatia, por rádio ou espírito?"). Estes itens que sugerem delírios de influência e perseguição e a alucinação auditiva estão relacionados a quadros psicóticos.

Tratamento $(\mathrm{EV}=1.599, \mathrm{VE}=4,5 \%)$. Este fator é formado basicamente pelas questões 35 ("Já utilizou ou usa atualmente remédio para dormir ou acalmar os nervos?") e 40 ("Recebe tratamento para nervosismo ou doença mental?"); em todas as análises, aparecem juntas, formando o fator Tratamento. A questão 35 também integra o fator Ansiedade/Somatização nas análises em Porto Alegre e na amostra total.

Ao fator Tratamento aparecem associadas à questão 2 ("Tem dificuldade para dormir?"), em Brasília; à questão 32 ("As Vezes fica muito tempo numa posição estranha?"), em São Paulo; aparece associada às questões 16 ("Não consegue trabalhar por nervosismo ou doença mental?") e 15 ("Já esteve descontrolado, fora de si, como se fosse doente da cabeça?"). em Porto Alegre; e à questão 24 ("Já sofreu um ataque depois de um susto ou contrariedade?"), na amostra total. Do ponto de vista psicopatológico as associações de tratamento com dificuldade de dormir, situações de descontrole, nervosismo, doença mental e quadros convulsivos são pertinentes. Mas chama a atenção que, enquanto fator, esta dimensão, não propriamente psicopatológica, tenha um comportamento relativamente independente dos outros quadros sintomatológicos.

Os fatores encontrados são compatíveis com os quadros psicopatológicos encontrados nas descrições originados da prática clínica; no entanto, devemos ressaltar que cada fator representa uma dimensão psicopatológica e não propriamente um diagnóstico. $O$ que temos como resultado das análises é a expressão do relacionamento entre atributos (sintomas, comportamentos e tratamentos), dentro de um contex to psiquiátrico, limitado pelo conteúdo das questões e pelas características $\mathrm{da}$ população investigada, enquanto que um diagnóstico implica uma classificação que é uma relação entre indivíduos (Kendell ${ }^{17}, 1975$ ). Esta diferença é importante, principalmente para entender e interpretar o significado destes fatores.

Além destas considerações estruturais e de conteúdo, os resultados da presente avaliação do QMPA permitem considerações a respeito do nível de medida dos diversos quadros psicopatológicos. Os níveis seriam o dimensional e o de categoria. Dimensional no sentido do quadro psi- 
copatológico poder ser medido de forma contínua, ou seja, utilizando o somatório das questões como escore, de forma que tanto maior será o comprometimento quanto maior o escore. $O$ de categoria, no sentido do quadro psicopatológico estar ou não presente, não permitindo medidas intermediárias.

Observando-se do primeiro ao sétimo fator é possível identificar um gradiente que vai de uma não - especificidade a uma especificidade crescente com relação ao quadro e gravidade do transtorno psicopatológico. Nos dois primeiros fatores, dependendo do número de questões positivas, $o$ indivíduo pode, desde ter um sintoma sem necessariamente ter transtornos psicopatológicos, até apresentar quadros de ansiedade, somatização, depressão e irritabilidade. Neste caso, podemos admitir que quanto mais questões o indivíduo apresentar nestes fatores, mais comprometido estará.

O terceiro fator, representado pela dimensão Deficiência Mental, é mais específico do que os fatores anteriores. Uma observação mais detalhada permite considerar que existe uma variação de graus de comprometimento que vai de um retardo mental leve a grave. Mesmo permitindo uma noção de gradiente, neste fator o que importa para diferenciar entre grau leve ou grave não é a quantidade de questões respondidas, mas sim quais delas estão agrupadas. Portanto, este fator tem um nível de medida categórico.

Do quarto fator em diante aumenta a especificidade, basta apresentar uma só questão positiva nestes fatores e o indivíduo pode ser considerado um "caso" (alcoolismo, quadros maníacos ou psicóticos). Isto revela um nível de medida categórico.

Kendell ${ }^{17}$ (1975) apresenta a discussão entre níveis de medida dimensional e categórico da doença mental, mostrando argumentos a favor $e$ contra, e sugerindo que existem fortes argumentos a favor da representação contínua da "doença neurótica" e dos transtornos de personalidade. Comenta também que existem fortes argumentos para considerar a "doença psicótica" como categórica. Os resultados encontrados apontam para essas observações. De qualquer modo, o presente trabalho não permite tais afirmações de modo satisfatório. Estas são especulações sobre a composição dos fatores encontrados. Argumentos a favor ou contra estas teorias utilizando os presentes dados seriam obtidas através de análises de validação de cada fator, comparando-os com os diversos diagnósticos psiquiátricos feitos de forma padronizada.

O baixo desempenho do QMPA para identificação de "caso" na comunidade, apresentado por Almeida-Filho e col.' (1992), muito provavelmente é decorrente do caráter específico de suas questões e da forma de medida utilizada, qual seja, o somatório das questões positivas com ponto de corte $7 / 8$. As principais escalas de rastreamento utilizam esta forma de medida com um ponto de corte estabelecido após uma validação. Entretanto o conteúdo de suas questões é mais geral e inespecífico para transtornos psicopatológicos do que o conteúdo das questões do QMPA. Nesse sentido, os presentes resultados apontam para questionários não específicos como sendo os melhores para identificação de "caso" na comunidade. O que está de acordo com as sugestões de D'Arcy ${ }^{6}$ (1982), que afirma que as questões que medem "bem estar social" têm papel importante na identificação de prováveis "casos" na comunidade.

\section{Conclusões e Recomendaçōes}

Em conclusão, o presente estudo verificou que o QMPA tem uma estrutura fatorial consistente. As mesmas dimensões psicopatológicas foram encontradas nas três cidades e na matriz total (representada pelo somatório das três matrizes). Estas dimensões mostram uma semelhança com a teoria dos quadros psicopatológicos descritos a partir de observações da prática clinica. Deste modo, foi possível interpretar cada uma das dimensões usando os modelos teóricos da psicopatologia.

$O$ QMPA revelou ser um questionário com dimensões bem definidas. As primeiras duas dimensões (Ansiedade/Somatização e Irritabilidade/ Depressão) sugerem uma estrutura psicopatologica ligada a transtornos neuróticos. Em termos psicométricos estes doi .uores sugerem um nível de medida dimensional. Os outros fatores encontrados sugerem um nível de medida categórico. Existe, entretanto, a necessidade de novos estudos no sentido de validar cada uma destas dimensões.

Os resultados alcançados sugerem que os questionários mais inespecíficos para transtornos mentais e as medidas de "bem estar social" são os mais adequados para a identificação de "caso" na comunidade.

ANDREOLI, S.B. et al. [The factor structure of the adult psychiatry morbidity questionnaire (QMPA) in a community sample of Brazilian cities]. Rev. Saúde Pública, 28: 249-60, 1994. Principal Components Analysis is a multivariate statistical technique for the purpose of examining the interdependence among variables. The main characteristic of this technique is the ability to reduce data, and it is currently used as an adjunct for the development of psychiatric research tools and the classification of psychiatric disorders. It has been applied to the study of the Factorial Structure of a Brazilian screening questionnaire, the Adult Psychiatric 
Morbidity Questionnaire (QMPA). The questionnaire is made up of 45 yes/no items for the identification of psychiatric symptons and the use of psychiatric services and psychotropic drugs. The questionnaire was applied to 6.470 subjects over 15 years old in representative samples from three urban areas: Brasília, Porto Alegre and S. Paulo. Seven factors were found to explain $42.7 \%$ of the total variance: Anxiety/Somatization (eigenvalue $=3.81,10.9 \%$ ); Irritability/Depression (eigenvalue $=2.41,6.9 \%$ ); Cognitive Impairment (eigenvalue $=2.01,5.8 \%$ ); Alcoholism (eigenvalue $=1.90,5.4 \%$ ); Mood Elation (eigenvalue $=1.62,4.6 \%$ ); Hallucinatory/ Delusional Disorders (eigenvalue $=1.60,4.6 \%$ ); and Drug/Therapies (eigenvalue $=1.60,4.5 \%$ ). A similar pattern of results was found when the analysis was carried out in the three places. It is suggested, on the banis of these findings, that some questions should be modified and some excluded in any future version of the questionnaire.

Keywords: Psychiatry, methods. Psychiatric status rating scales. Factor analysis, statistical.

\section{Referências Bibliográficas}

1. ALMEIDA FILHO, N. de et al. Estudo multicêntrico de morbidade psiquiátrica e neurológica em áreas urbanas brasileiras (Brasíia, São Paulo, Porto Alegre) - I: Metodologia e resultados preliminares. Revista $A B P$ APAL, 14:93-104, 1992.

2. AMERICAN PSYCHIATRIC ASSOCIATION (APA). Diagnostic and statistical manual of mental disorders. 3rd ed. Washington, 1980.

3. BMDP STATISTICAL SOFTWARE. BMDP statistical software manual. University of California Press, 1990.

4. BURVILL, P.W. \& KNUIMAN, M.W. Which Version of the General Health Questionnaire should be used in community studies? Australian \& New Zealand Journal of Psychiatry, 17:237-42, 1983.

5. CARMINES, E.G. \& ZELLER, R.A. The place of factor analysis in reliability and validity assessment. In: Reliability and validity assessment. Beverly Hills, Sage Publication, 1979. p. 59. (Sage University Papers Series on Quantitative Applications in the Social Sciences).

6. D'ARCY, C. Prevalence and correlates of nonpsychotic psychiatric symptoms in the general population. Can. J. Psychiatr., 27:316-24, 1982.

7. DOHRENWEND, B.P. et in. Mental illness in the United States-Epidemiological Estimates. New York, Praeger, 1980.
8. DOHRENWEND, B.P. et al. Nonspecific psychological distress and other dimensions of psychopathology. Arch. Gen. Psychiatr. 37:1229-36, 1980.

9. GOLDBERG, D. The detection of psychiatric illness by questionnaire. London, Oxford University Press, 1972.

10. GOLDBERG, D. Manual of the general health questionnaire. London, NFER Publishing Company, 1978.

11. GOLDBERG, D. \& WILLIAMS, P. A user's guide to the general health questionnaire. London, NFER Publishing Company, 1990.

12. GROOT, H.R. \& AREVALON, W. Validez de un instrumento para detectar enfermedad mentul. Cali, Universi. dad del Valle, s.d.

13. JOHNSON, R. \& WICHERN, D.E. Applied multivariate statistical analysis. 2nd ed. Englewood Cliffs, Prentice Hall, 1988. p.380-437: factor analysis and inference for structured covariance matrices.

14. KAISER, H.F. The varimax criterion for analytic rotation in factor analysis. Psychometrika, 23:187-210, 1958.

15. KAISER, H.F. The application of electronic computers to factor analysis. Educ. Psvchol. Measur., 20:141-51, 1960.

16. KAPLAN, H.I. \& SADOCK, B.J. Compêndio de Psiquiatria. 2 ed. Porto Alegre, Artes Médicas, 190, 1990.

17. KENDELL, R.E. The role of multivariate analysis in deriving or validating classifications. In:The role of diagnosis in psychiatry. Oxford, Blackwell Scientific Publications, 1975. p.106-18.

18. LAURA, E. El universo multivariado y su analisis. In:Analysis multivariado: metodo de componentes principales. Washington, D.C. Secretaría General de la Organización de los Estados Americanos, Programa Regional de Desarollo Científico y Tecnológico, 1986. p.3-10:

19. RAMOS, L. \& GOIHMAN, S. Geographical stratification by socio-economic status: methodology from household survey with elderly people in São Paulo, Brazil. Rev. Saúde Pública, 23:479-92, 1989.

20. SANTANA, V. Estudo epidemiológico das doenças mentais em um bairro de Salvador. Salvador, Instituto de Saúde do Estado da Bahia, 1982. (Série Estudos em Saúde, 3).

21. UNIVERSIDADE DE SÃO PAULO. Faculdade de Medicina. Departamento de Medicina Preventiva. Pesquisa na área de saúde mental; relatório preliminar. São Paulo, 1977.

22.VÁZQUEZ-BARQUERO, J.L. et al. The factor structure of the GHQ-60 in a "community sample. Psychol. Med., 18:211-8, 1988.

23. WEISS, D.J. Factor analysis and counseling research. $J$. Counseling Psychol., 7:477-85, 1970.

Recebido para publicação em 13.1.1994 Reapresentado em 3.5.1994

Aprovado para publicaçāo em 7.7.1994 